\title{
Myricetin Prevents Fibrillogenesis of Hen Egg White Lysozyme
}

Jianwei He, ${ }^{* \dagger}{ }^{\dagger}$ Yu Wang, ${ }^{\dagger}$ Alan K. Chang, ${ }^{\dagger}$ Linan $\mathrm{Xu},{ }^{\dagger}$ Na Wang, ${ }^{\dagger}$ Xiaoying Chong, ${ }^{\dagger}$ Hui Li, ${ }^{\dagger}$ Bing Zhang,

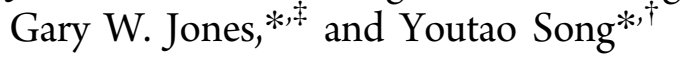

${ }^{\dagger}$ Province Key Laboratory of Animal Resource and Epidemic Disease Prevention, School of Life Science, Liaoning University, Shenyang 110036, China

${ }^{\ddagger}$ Department of Biology, National University of Ireland Maynooth, Maynooth, County Kildare, Ireland

${ }^{\S}$ Experimental Center of Functional Subjects, China Medical University, 92 Bei Er Road, Heping District, Shenyang 110001, China

ABSTRACT: Myricetin is a natural flavonol found in many grapes, berries, fruits, vegetables, and herbs as well as other plants. Recent studies have identified potential antiamyloidogenic activity for this compound. In this study, the kinetics of amyloid fibril formation by hen egg white lysozyme (HEWL) and the antifibril-forming activity of myricetin were investigated. We demonstrate that myricetin significantly inhibits the fibrillation of HEWL and the inhibitory effect is dose-dependent. Interestingly, the inhibitory effect toward HEWL fibrillation was stronger than that exerted by the previously characterized fibril-forming inhibitor quercetin, which has high structural similarity with myricetin. Spectrofluorometric and computational studies suggest that the mechanism underlying the inhibitory action of myricetin at a molecular level is to reduce the population of partially unfolded HEWL intermediates. This action is achieved by the tight binding of myricetin to the aggregation-prone region of the $\beta$-domain of HEWL and linking to the relatively stable $\alpha$-domain, thus resulting in the inhibition of amyloid fibril formation.

KEYWORDS: myricetin, lysozyme, amyloid, molecular dynamics simulation, molecular docking

\section{INTRODUCTION}

Amyloid diseases are characterized by conformational changes of certain proteins, which lead to intracellular or extracellular aggregation, eventually resulting in fibrillar amyloid deposits in the affected tissue. Currently there are approximately 30 extracellular fibril proteins known to be associated with human amyloidosis. ${ }^{1}$ The so-called "hereditary non-neuropathic systemic amyloidosis" is associated with two lysozyme variants (I56T and D67H), which were identified in the early $1990 \mathrm{~s}^{2}$ and other naturally occurring amyloidogenic variants (F57I, F57I/ T70N, W64R, and T70N/W112R) discovered more recently. ${ }^{3-5}$ The pathological characteristics of these amyloidoses includes the deposition of the full-length protein variants in organs, such as liver, spleen, and kidneys. ${ }^{2}$

At present there are still no effective treatments for amyloid diseases such as Alzheimer's disease, systemic amyloidosis, familial amyloid polyneuropathy, and Creutzfeldt-Jakob disease, and all drugs in development that target Alzheimer's disease have failed at various stages of clinical trials. ${ }^{6}$ However, the identification and development of small molecules that inhibit fibril formation by amyloidogenic proteins is a promising area of research. ${ }^{7,8}$ Among the most promising small-molecule inhibitors, the naturally occurring polyphenols show the most effective antiamyloidogenic activity. 9 A group of polyphenols found in green tea (GTPs) including (-)-epigallocatechin (EGC), (-)-epicatechin gallate (ECG), and (-)-epigallocatechin gallate (EGCG) have been reported as effective inhibitors of alkali-saltinduced fibrillogenesis of hen egg white lysozyme (HEWL) with ECG as the most potent polyphenol. ${ }^{10} \mathrm{~A}$ previous report demonstrated that curcumin can also inhibit the aggregation and formation of amyloid fibrillation of HEWL at $\mathrm{pH} 2.0$ in a dosedependent manner. ${ }^{11}$ In addition, some other small molecules have been demonstrated to show inhibitory effects on HEWL amyloid fibril formation, such as acridine derivatives ${ }^{12-14}$ and carnosine-a common dipeptide in mammals. ${ }^{15}$ However, the relative inhibitory effects between these small molecules are difficult to compare systematically because of varied experimental conditions including temperature, concentration of protein, $\mathrm{pH}$, and rotation speeds, applied by different research groups. Along with relatively well characterized polyphenols such as ECGC and curcumin, myricetin has also recently been investigated for antiamyloidogenic activity (Figure 1). ${ }^{16}$ Myricetin has been shown to exhibit stronger inhibitory activity against $\mathrm{A} \beta$ fibrillation compared to other flavonoids of similar structures, including quercetin and luteolin, both of which<smiles>O=c1c(O)c(-c2cc(O)c(O)c(O)c2)oc2cc(O)cc(O)c12</smiles>

3,5,7-Trihydroxy-2-(3,4,5-trihydroxy-phenyl)-chromen-4-one (myricetin, flavonol)

Figure 1. Chemical structure of myricetin.

Received: May 28, 2014

Revised: August 8, 2014

Accepted: September 7, 2014

Published: September 8, 2014 
possess fewer hydroxyl groups on the B ring. ${ }^{17}$ Although the detailed molecular mechanisms underlying the inhibitory effects of flavonoids remain unclear, these results suggest that differences in hydroxyl groups contained within the flavonoid structure may influence the formation of hydrogen bonds between $\mathrm{A} \beta$ and its hydroxyl arms, and is possibly the key determinant of antiamyloidogenic activity. ${ }^{17}$

Many of the currently investigated small-molecule inhibitors act as general antiamyloid agents, which affect a range of amyloidogenic proteins. ${ }^{18-20}$ Although the property of nonspecificity is typically not desirable for drug design, it can offer the possibility of broad-spectrum therapeutics, but only provided there is no effects on functional human fibrous proteins such as those that constitute the cytoskeletal system. Currently myricetin has been demonstrated as exhibiting antiamyloidogenic activity against A $\beta, \alpha$-synuclein, IAPP. ${ }^{17,21,22}$ We sought to assess further the general nature of inhibitory activity by investigating whether myricetin could inhibit fibril formation in vitro of hen egg white lysozyme. HEWL has two domains, $\alpha$ and $\beta$, and each consists of four $\alpha$-helices and an antiparallel $\beta$-sheet. Its structure is highly homologous to human lysozyme, which is responsible for the formation of systemic amyloidosis. ${ }^{23}$

In this study, thioflavin $\mathrm{T}$ ( ThT) fluorescence assays and transmission electron microscopy (TEM) were used to detect and assess the inhibitory activity of myricetin upon fibril formation by HEWL. Additionally, the interaction between myricetin and the monomeric form of HEWL was investigated using a combination of anilinonaphthalene-8-sulfonic (ANS) and intrinsic fluorescence assays coupled with in silico simulations.

\section{MATERIALS AND METHODS}

Proteins and Reagents. HEWL was purchased from Solarbio (Beijing, China). ThT, ANS, myricetin, and quercetin were purchased from Sigma-Aldrich (St. Louis, MO, USA).

HEWL Sample Preparation. A stock solution of myricetin (50 $\mathrm{mM}$ ) was prepared in dimethyl sulfoxide (DMSO). HEWL sample solutions were prepared by dissolving $1 \mathrm{mg}$ of HEWL powder in $1 \mathrm{~mL}$ of $50 \mathrm{mM}$ glycine-hydrochloric acid buffer $(\mathrm{pH} 2.0)$ with or without an aliquot of myricetin solution. The final concentration of DMSO in HEWL sample solution is $0.2 \%$, of which we confirmed that $0.2 \%$ DMSO had no obvious effect on fibrillar structure and ThT fluorescence. Incubation was carried out with constant agitation at $140 \mathrm{rpm}$ at $65^{\circ} \mathrm{C}$ during the course of aggregation.

Thioflavin T Fluorescence Assay. Phosphate buffer $(50 \mathrm{mM}$ $\mathrm{Na}_{2} \mathrm{HPO}_{4}, 50 \mathrm{mM} \mathrm{NaH} \mathrm{PO}_{4}, \mathrm{pH}$ 7.0) was used to dissolve ThT to a stock concentration of $1 \mathrm{mM}$. Aliquots of HEWL samples taken at different times were diluted with phosphate buffer ( $\mathrm{pH} 7.0)$, followed by the addition of an aliquot ( $30 \mu \mathrm{L}$ stock solution) of ThT stock solution. $\mathrm{ThT}$ fluorescence measurement was conducted by exciting the sample at $440 \mathrm{~nm}$ and recording the emission intensity at $485 \mathrm{~nm}$ over $120 \mathrm{~s}$. The measurement was carried out using a Cary Eclipse fluorescence spectrophotometer (Varian, Palo Alto, CA, USA).

Intrinsic Fluorescence Assay. Steady state intrinsic fluorescence spectrum was measured with a Cary Eclipse fluorescence spectrophotometer (Varian) using a quartz cuvette with a path length of $1 \mathrm{~cm}$. HEWL samples $(30 \mu \mathrm{L})$ with or without myricetin were diluted to a final volume of $3 \mathrm{~mL}$ and then subjected to analysis. The sample was excited at $280 \mathrm{~nm}$, and the spectrum was recorded between 300 and $500 \mathrm{~nm}$.

ANS Fluorescence Assay. A stock solution of ANS (0.4 mM) was prepared by dissolving ANS in phosphate-buffered saline (PBS; $\mathrm{pH}$ 7.0). The ANS stock solution was stored at $4{ }^{\circ} \mathrm{C}$. Aliquots of HEWL solution with or without myricetin taken at different times were mixed with an aliquot of ANS solution, followed by the addition of PBS (pH7.0) to a final of volume of $3 \mathrm{~mL}$. After incubation in the dark for $30 \mathrm{~min}$ at room temperature, the samples were subjected to fluorescence assay using a
Cary Eclipse fluorescence spectrophotometer (Varian). The samples were excited at $380 \mathrm{~nm}$, and fluorescence was recorded between 400 and $600 \mathrm{~nm}$. Both the ANS fluorescence intensity and the average emission wavelength were recorded to account for the changes in intensity and spectrum. We confirmed that myricetin had no obvious effects on ThT, ANS, and intrinsic fluorescence.

Transmission Electron Microscopy. HEWL samples with and without myricetin were diluted 5-fold, and $10 \mu \mathrm{L}$ of each sample was dispensed onto a coated copper-mesh grid. The grid was negatively stained with $1 \%(\mathrm{w} / \mathrm{v})$ phosphotungstic acid and then observed with a Hitachi H-7650 transmission electron microscope with an accelerating voltage of $80 \mathrm{kV}$.

Molecular Dynamic Simulations and Docking Studies. All molecular dynamics (MD) simulations were carried out using the GROMACS 4.0.7 software package. ${ }^{24}$ The crystal structure of HEWL (PDB entry 1GXV) was downloaded from the Protein Data Bank (PDB). ${ }^{25}$ The GROMOS96 $43 \mathrm{al}^{26}$ force field was adopted in all simulations in this study. The monomer model was solvated in a water box containing 10,124 simple point-charge water molecules ${ }^{27}$ and neutralized by adding eight $\mathrm{Cl}^{-}$counterions. The linear constraint solver method $^{27}$ was used to constrain the bond length, allowing for integration time steps of $2 \mathrm{fs}$ throughout the whole simulation. Electrostatic interactions were calculated with the particle-mesh Ewald algorithm. ${ }^{28}$ A cutoff of $9 \AA$ was used for all calculations. The solvated and neutralized systems were then energy-minimized for 20,000 steps to remove bad contacts. Then the side chains and solvent were allowed to move unrestrained for $40 \mathrm{ps}$, with the backbone atoms of the structure fixed. To investigate the structural fluctuation of HEWL under amyloid fibril forming conditions, $\mathrm{MD}$ simulations at $338 \mathrm{~K}$ and $\mathrm{pH} 2.0$ were carried out. For the control, a physiological condition of $310 \mathrm{~K}$ and $\mathrm{pH}$ 7.0 was used. After equilibration, two independent simulations were carried out for $20 \mathrm{~ns}$.

Docking studies were performed using Autodock 4.2.5.1. The 3D structure of myricetin was obtained from TCM Database@Taiwan. ${ }^{29}$ Prior to autodocking the HEWL molecule and myricetin were prepared using AutoDockTools 1.5.4, including addition of polar hydrogen atoms and setting the charges of HEWL residues using Kollman charges and adding Gasteiger charges to myricetin.

The docking grids were carried out with a default spacing value of $0.375 \AA$ and a grid volume of $60 \times 60 \times 60$ points covering the entire surface of HEWL. The protein molecule was set to be rigid while the ligand molecule was considered to be flexible. In the docking simulation, a Lamarckian geneti algorithm was used to generate 50 binding models, each of which was individually selected as the lowest binding energy (calculated using the Autodock scoring function) among a maximum of 2,500,000 evaluations. These 50 complexes were clustered according to their root-mean-square deviation (RMSD) values and binding energies in order to distinguish the preferred binding model.

Statistical Analysis. All experiments were performed at least three times, and all data are presented as means \pm standard deviations (SDs). Comparisons between groups were tested by one-way ANOVA analysis. Statistical significances of data were considered at the $P<0.05$ level.

\section{RESULTS}

Analysis of HEWL Fibril Formation Using ThT Fluorescence. HEWL is a model protein for amyloid research as it is known to readily form fibrils under conditions of low $\mathrm{pH}$ and elevated temperature, when coupled with mechanical agitation. ${ }^{30}$ By monitoring the kinetics of HEWL fibril formation by fluorescence spectroscopy, we found that the relative fluorescence of HEWL increased with extended incubation times (Figure 2A), which is in agreement with previous studies. ${ }^{31}$ The consistent increase in ThT fluorescence intensity observed when HEWL was incubated alone, demonstrates that formation of amyloid fibrils proceeded rapidly and without a lag phase (Figure $2 A)$. The inhibitory effect exerted on the formation of HEWL fibril by the addition of myricetin $(100 \mu \mathrm{M})$ into the assay resulted in a strong inhibition of HEWL fibril formation (Figure 


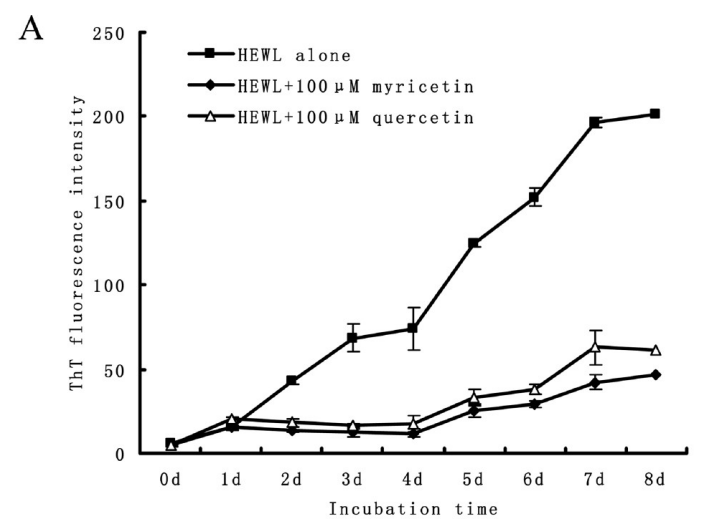

B

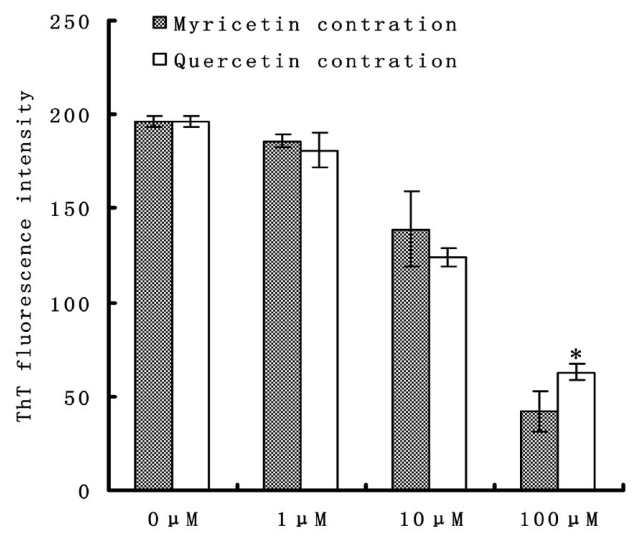

$\mathrm{C}$

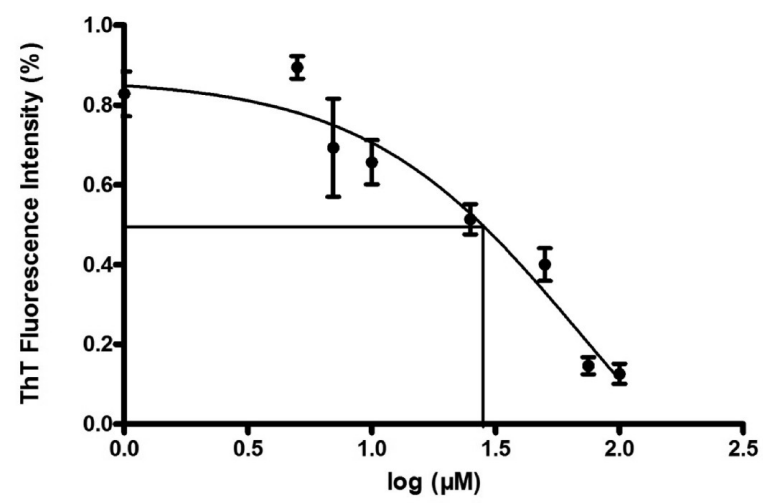

Figure 2. Effects of myricetin and quercetin on amyloid formation by HEWL: (A) the kinetics of HEWL fibril formation in the absence (filled squares) or the presence of myricetin (filled diamonds) or quercetin (filled upward triangles) as monitored by ThT fluorescence; (B) dosedependent inhibitory capacity of myricetin and quercetin on amyloid formation by HEWL 7 days after incubation; (C) dose-response curve plotting the plateau value of the ThT fluorescence intensity of the HEWL sample against myricetin concentration.

2A). Compared to quercetin-containing HEWL samples (the positive control group), myricetin showed a reproducible moderately stronger inhibition.

To determine an effective concentration range of myricetin for inhibiting HEWL fibril formation, varying aliquots of myricetin were mixed with HEWL to final concentrations of 1,10 , and 100 $\mu \mathrm{M}$; at these concentrations we did not observe significant toxic effects of myricetin on cell survival (data not shown) and the ThT fluorescence intensity measured (Figure 2B). Myricetin (and quercitin control) was observed to exhibit a dosedependent effect on HEWL fibril formation (Figure 2B).
Although fibril inhibition was observed up to a concentration of $10 \mu \mathrm{M}$, major inhibitory effects were only observed at a concentration of $100 \mu \mathrm{M}$, indicating effective fibril inhibition occurs between these two concentrations. Importantly, at 100 $\mu \mathrm{M}$ the inhibitory effect of myricetin on HEWL fibril formation was significantly stronger $(p$-value $=0.037626<0.05)$ than that previously obtained with quercetin (Figure $2 \mathrm{~B}$ ), the positive control.

By calculating the percentage of protection from the ThT curve of HEWL alone and fitting a logarithmic concentrationresponse curve, myricetin inhibited HWEL fibril formation with a median effective concentration (IC50 value) of $28.2 \mu \mathrm{M}$ (Figure 2C).

Assessing Structural Changes during HEWL Fibril Formation. ANS is a fluorescencent dye that has high affinity for the hydrophobic surfaces of proteins. Upon binding to the hydrophobic regions of a protein surface, the intensity of the light emission is increased, with the emission maximum showing a blue shift. ${ }^{32}$ This technique allows changes in the hydrophobic core and the stability of a whole protein to be monitored during the course of aggregation. As shown in Figure 3A, a significant
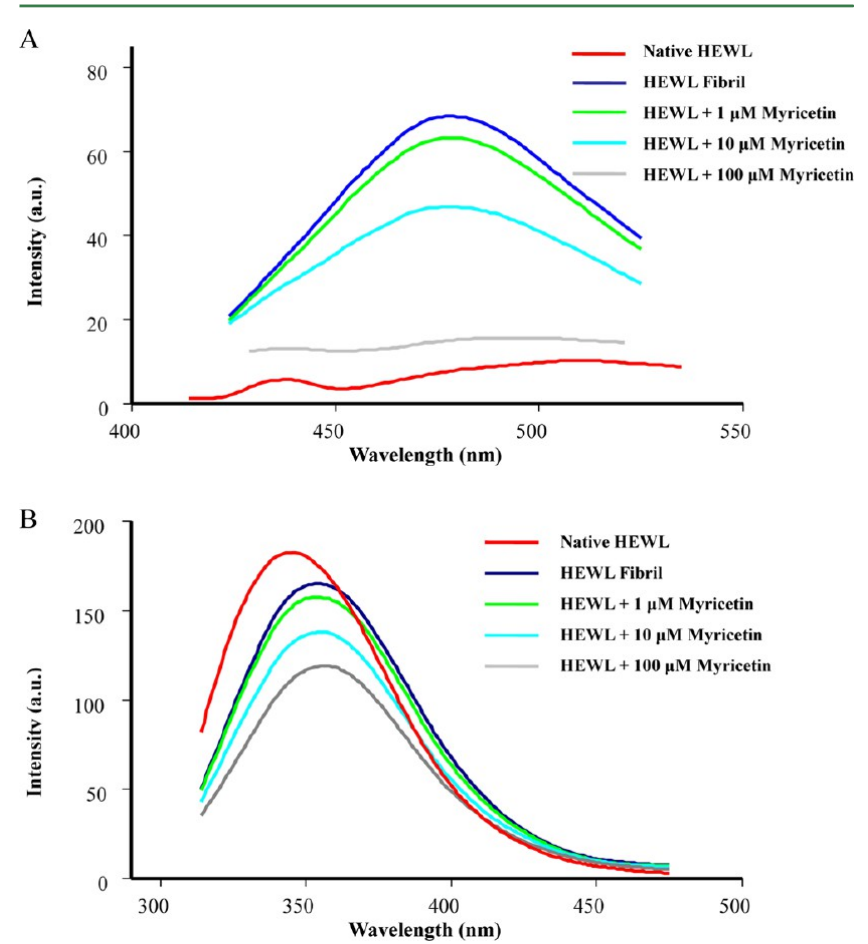

Figure 3. (A) ANS fluorescence emission spectra of HEWL fibril incubated with (pink) or without (red) myrecetin after incubation for 7 days. (B) Intrinsic fluorescence emission spectra of HEWL fibril in the absence (red) and presence (purple) of myricetin after incubation for 7 days.

blue shift from 515 to $473 \mathrm{~nm}$ and increase in intensity of ANS fluorescence, compared to native HEWL, is caused by HEWL fibril formation. The addition of $100 \mu \mathrm{M}$ restores HEWL fibril ANS intensity to levels comparable with the native protein.

Variation in the microenvironment of tryptophan residues and monitoring the intrinsic fluorescence of a protein allow assessment of structural characteristics. ${ }^{33}$ To further probe the effect of myricetin on the conformation of HEWL, the intrinsic fluorescence of HEWL in the absence or presence of myricetin was measured. A decreased intensity in intrinsic fluorescence of 
HEWL fibril group with a shift from 344 to $355 \mathrm{~nm}$ was observed compared to the native HEWL group (Figure 3B). This indicated that tryptophan in HEWL became more accessible to solvent during the process of fibril formation, resulting in the quenching of fluorescence by water. ${ }^{34,35}$ However, the presence of myricetin significantly reduced the fluorescence intensity during fibril formation (Figure 3B). The quenching of intrinsic fluorescence may possibly result from the interactions between aromatic residues of myricetin and the aromatic residues of target proteins. $^{36,37}$

Physical Analysis of HEWL Fibril formation. Direct information on the formation of fibril by HEWL as well as by the antifibrillogenic activity of myricetin was obtained using TEM. The morphology of HEWL fibers and/or aggregates in the absence or presence of myricetin is shown in Figure 4A-G. In the absence of myricetin or quercetin, HEWL displayed straight and regular premature or mature amyloid fibril structures with typical diameters ranging from 2 to $20 \mathrm{~nm}$ and several micrometers in length (Figure 4A,E). The presence of quercetin (Figure 4B) clearly resulted in reduced amounts of HEWL fibrils. In contrast, the presence of myricetin resulted in formation of amorphous aggregates, with virtually no HEWL fibrils being observed (Figure 4C). This result provides further evidence that myricetin exhibits a stronger inhibition against fibril formation by HEWL compared to quercetin. To elucidate the physical nature of myricetin fibril inhibition, by using TEM we also examined HEWL fibril formation and myricetin inhibition with prolonged incubation time and found $25 \mu \mathrm{M}$ myricetin (close to IC50 value) incubated with HEWL resulted in a reduced amount of amyloid fibril after 12 days incubation (Figure 4G). In contrast, the presence of $100 \mu \mathrm{M}$ myricetin resulted in formation of amorphous aggregates, with virtually no HEWL fibrils being observed (Figure 4D). Taken together, we concluded that the physical nature of fibril inhibition is determined by the concentration of myricetin. We also confirmed that the TEM images of myricetin at $100 \mu \mathrm{M}$ show only the deposition of the compound alone and no fibril structure was found (Figure 4H).

Computational Analysis of HEWL Fibril Formation. Based on the spectrometric and electron microscopy data, it can be inferred that myricetin inhibition of amyloid fibrils formation of HEWL mainly occurs at a very early stage in the process. To better understand the process of amyloid fibril formation by HEWL as well as the interaction of myricetin with lysozyme at the atomic level, we carried out MD simulations (see Materials and Methods).

Figure 5A compares the degree of conformational changes that occur in HEWL under two different MD simulation conditions, one favoring amyloid fibril formation $(338 \mathrm{~K}$ and $\mathrm{pH} 2.0)$ and the other under normal cellular physiological parameters $(310 \mathrm{~K}$ and $\mathrm{pH}$ 7.0). The backbone RMSD trajectory in fibril-forming condition (represented by the red trace) has a higher value, indicating a less stable state than that in physiological conditions (black trace). The root-mean-square fluctuation (RMSF) of each residue (Figure 5B) revealed a major contribution of the $\beta$ domain (residues from 36 to 87 ) toward the instability of HEWL under fibril-forming conditions. This is consistent with the dramatic changes of $\beta$-sheet content in the $\beta$-domain observed during the $20 \mathrm{~ns} \mathrm{MD}$ simulation, particularly the major difference in stability observed after $15 \mathrm{~ns}$ compared to changes seen under physiological conditions (Figure 5C). In contrast, the $\alpha$-domain maintained much of the native conformation with the content of $\alpha$-helix being constant (data not shown). From the superposition of the final conformational state following the $20 \mathrm{~ns} \mathrm{MD}$
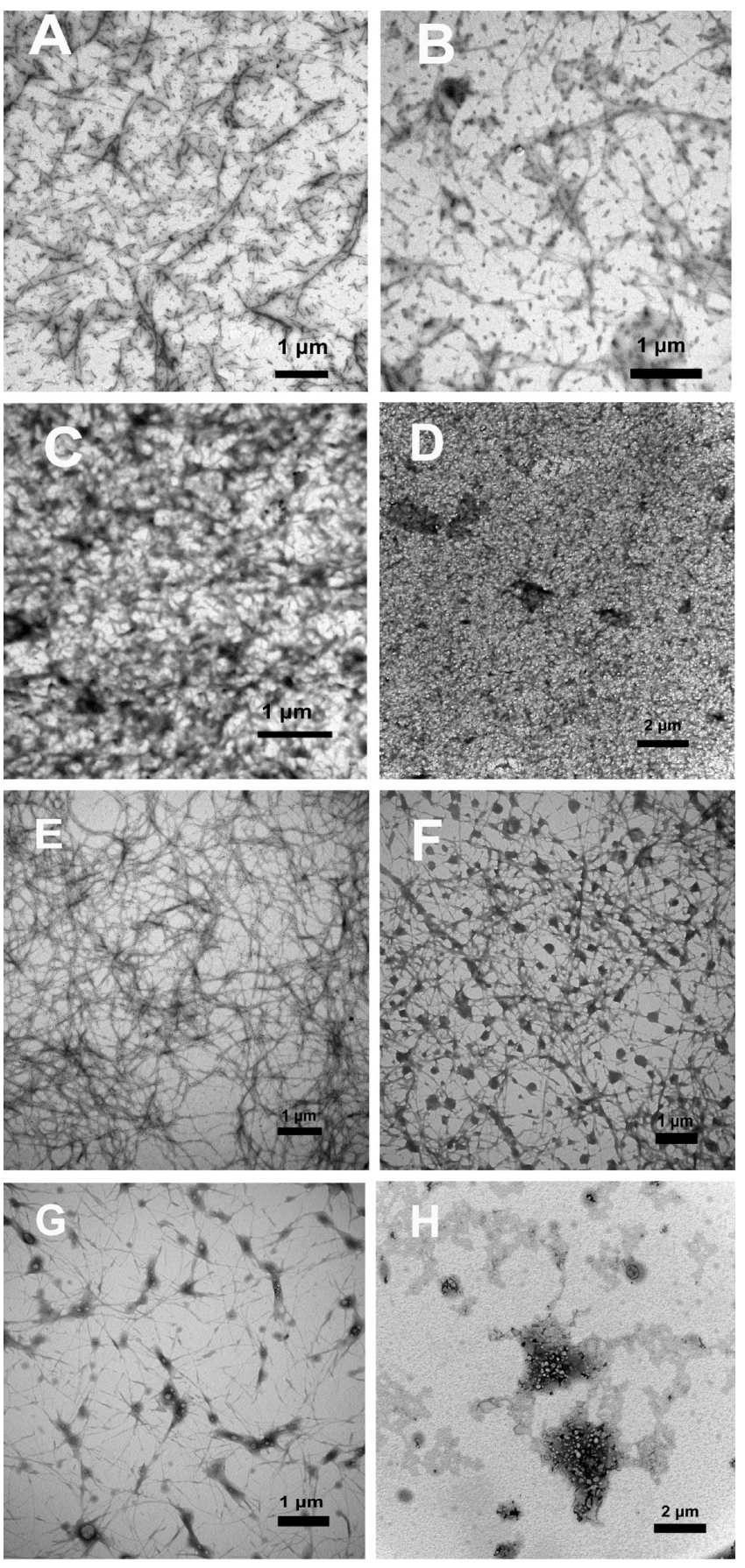

Figure 4. Transmission electron microscopic images of HEWL fibrils formed in the absence or presence of myricetin or quercetin, after incubation at $65^{\circ} \mathrm{C}$ and $\mathrm{pH} 2.0$ for 7 days or 12 days: (A) HEWL fibril after incubation for 7 days; (B) HEWL fibril in the presence of $100 \mu \mathrm{M}$ quercetin; (C) images of HEWL in the presence of $100 \mu \mathrm{M}$ myricetin after incubation for 7 days; (D) images of HEWL in the presence of 100 $\mu \mathrm{M}$ myricetin after incubation for 12 days; (E) HEWL fibril after incubation for 12 days; (F) images of HEWL in the presence of $10 \mu \mathrm{M}$ myricetin after incubation for 12 days; (G) images of HEWL in the presence of $25 \mu \mathrm{M}$ myricetin after incubation for 12 days; $(\mathrm{H})$ images of $100 \mu \mathrm{M}$ myricetin after incubation for 12 days. Scale bars represent 1 and $2 \mu \mathrm{m}$.

simulation, the clear disruption in the $\beta$-domain could be directly observed (Figure 5D).

To investigate how myricetin may affect the conformation of HEWL, we carried out docking simulations between these two 
A

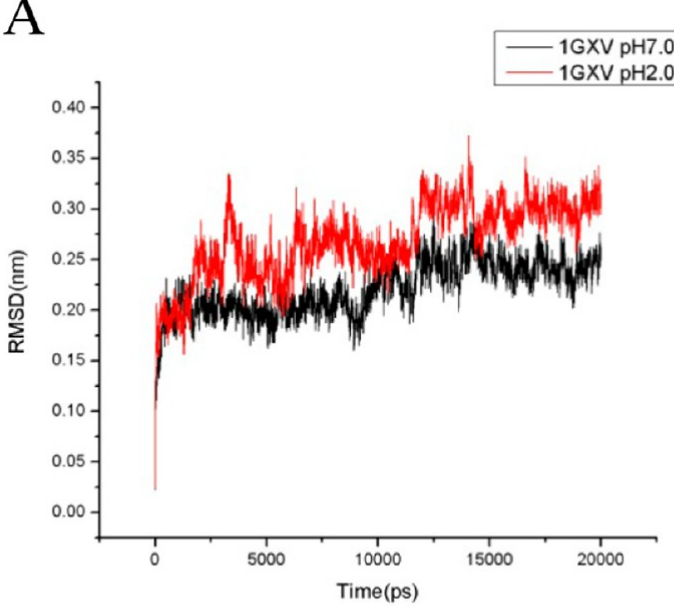

$\mathrm{C}$

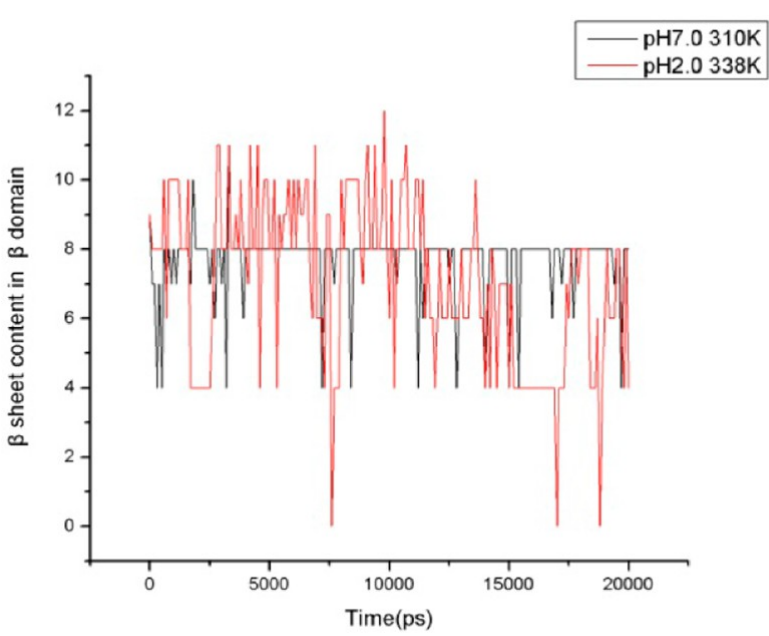

B

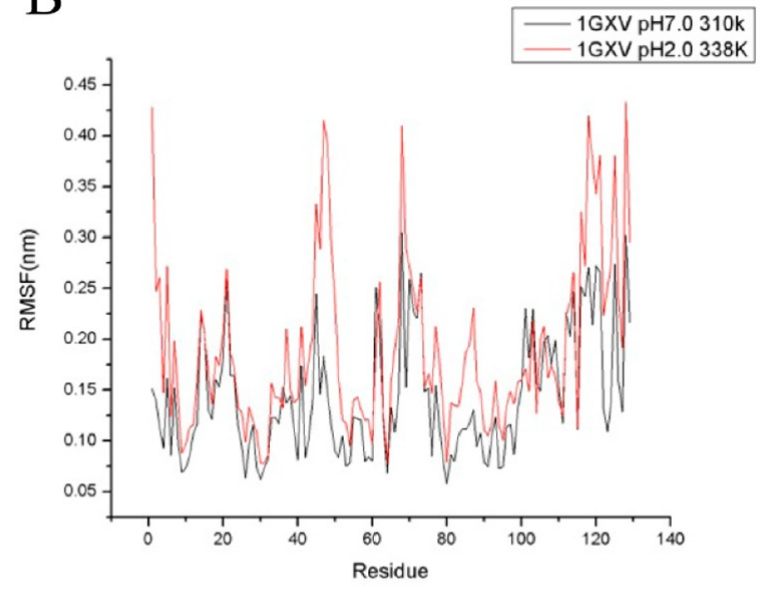

$\mathrm{D}$

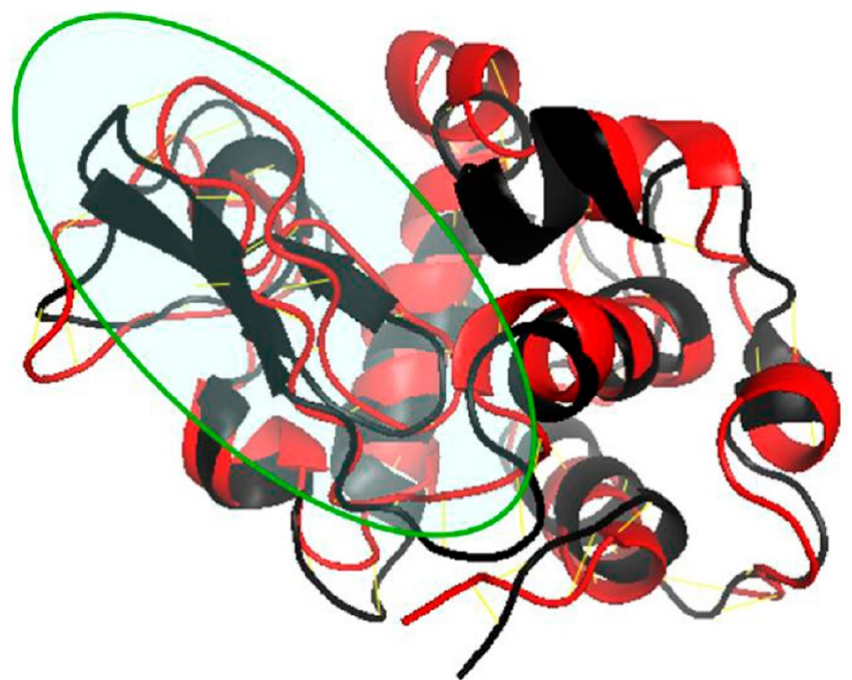

Figure 5. (A) RMSD of the C $\alpha$-positions during the 20 ns simulations. (B) RMSF trajectory per residue during the period of MD simulations. (C) Fluctuation of $\beta$-sheet content in $\beta$-domain through the $20 \mathrm{~ns} \mathrm{MD} \mathrm{simulation.} \mathrm{(D)} \mathrm{Superposition} \mathrm{of} \mathrm{the} \mathrm{final} \mathrm{state} \mathrm{of} \mathrm{the} 20 \mathrm{~ns}$ MD simulation at physiological condition (black) and amyloid fibril forming condition (red).

molecules. According to the docking results, myricetin was located in an area between the $\alpha$-domain and the $\beta$-domain (Figure 6A). The predicted binding site for myricetin on HEWL was clustered around many residues, such as Asn44, Asn46, Asp52, Asn59 and Glu35, Ala107, Val109, and Trp62 and Trp63. The interactions between myricetin and these residues were through van der Waals interactions and hydrogen bonding. Of particular note are the interactions with residues Trp62 and Trp63 in the $\beta$-domain, which could explain the quenching of intrinsic fluorescence (Figure 6B).

\section{DISCUSSION}

Amyloid diseases are either sporadic or familial in their nature. Usually, in the sporadic forms of the diseases the aggregated peptides/proteins display wild-type sequences, while in rarer early onset familial forms of the diseases the aggregation results from genetic mutations that exhibit a greater propensity for aggregation. ${ }^{38} \mathrm{~A}$ major risk factor for most sporadic amyloid diseases is aging. ${ }^{39}$ In older people, the age-related decline in cellular proteostasis includes the unfolded protein response, the ubiquitin proteasome system, autophagy, and the encapsulation of damaged proteins in aggresomes, all of which lead to the accumulation of protein aggregates. ${ }^{40-43}$ Progression of the aging process means that most kinds of amyloid diseases occur undetected in their early stages. However, an increasing number of research investigations suggest that the intake of diets containing natural small molecules with inhibitory activities toward amyloid fibril formation may help to prevent such diseases or at least delay early onset. ${ }^{44-46}$

Myricetin is a cell-permeable flavanoid often used in inflammatory, diabetes, and cancer studies, since it has a variety of pharmacological properties such as antioxidant activity and an ability to reduce blood sugar levels. ${ }^{47,48}$ High-throughput screens for small molecules exhibiting antiamyloidogenic activity demonstrated that myricetin could prevent amyloid fibril formation of A $\beta, \alpha$-synuclein, and IAPP. To further develop and characterize myricetin as a potential antiamyloid therapeutic agent, we assessed the ability of this small molecule to inhibit amyloid fibril formation by the model protein HEWL.

The inhibitory effect exerted by myricetin on HEWL fibril formation, as assessed by ThT fluorescence, was greater than the well-characterized polyphenol quercetin (Figure 2A). However, 
A
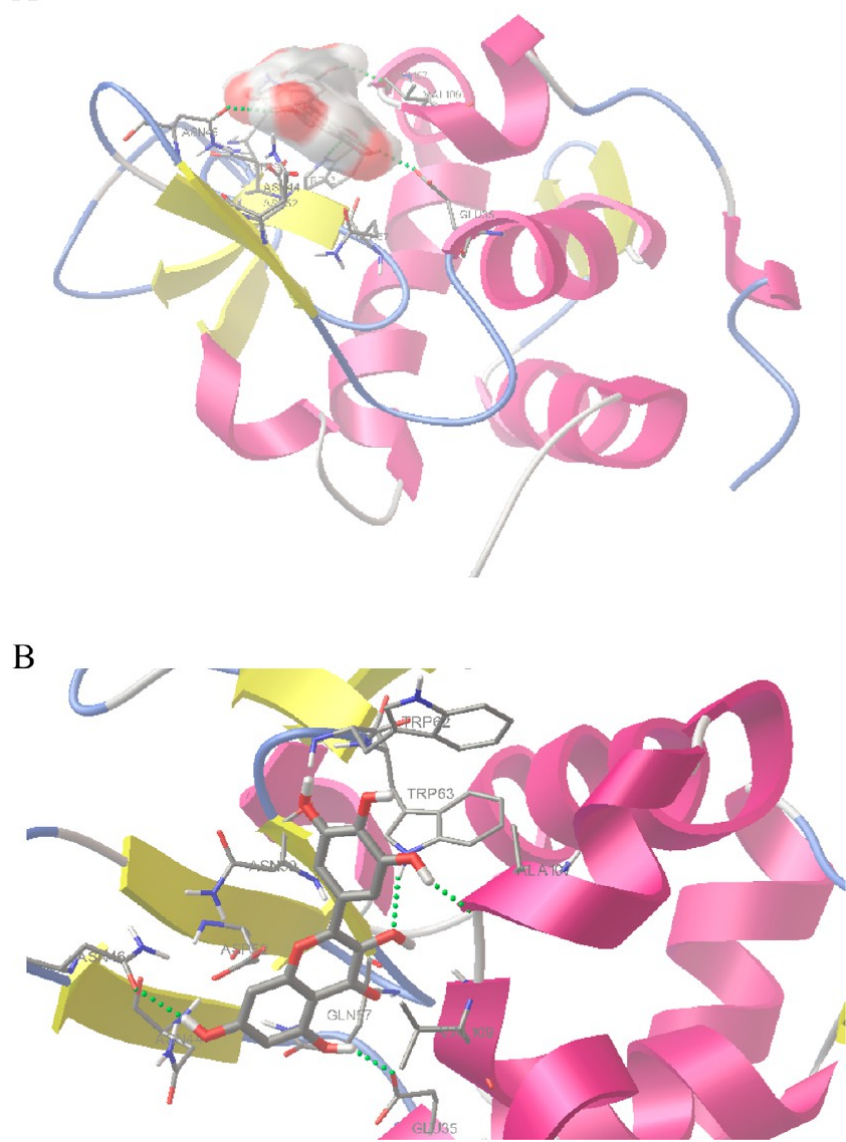

Figure 6. (A) Panoramic view showing the mode of binding between myricetin and HEWL. (B) Close-up view of the complex showing the binding between myricetin and HEWL, with hydrogen bonding marked as a green dotted line.

since ThT fluorescence induction can be biased by the presence of exogenous compounds, ${ }^{49}$ we also assessed the physical nature of myricetin fibril inhibition using TEM. Incubation of myricetin with HEWL resulted in the formation of amorphous aggregates, with little or no fibrils being observed (Figure 4C,D). This was in contrast to incubation of HEWL in the presence of quercetin, which resulted in a reduction of amyloid fibril formation, but no formation of amorphous aggregates (Figure 4B). The results from the TEM analysis therefore are in agreement with the ThT assay and clearly demonstrate that myricetin inhibits HEWL fibril formation more effectively than quercetin. While these experiments reveal the inhibitory potential of myricetin against amyloid fibrils, they do not provide any information regarding the potential molecular mechanisms underlying this activity. In an attempt to gain insight into the molecular nature of fibril inhibition we turned to well-developed computational approaches.

Previous studies demonstrated that amyloidogenic variants of human lysozyme such as $\mathrm{I} 56 \mathrm{~T}$ and $\mathrm{D} 67 \mathrm{H}$, undergo transient and local cooperative unfolding in the $\beta$-domain region of the protein, a process that occurs at the early stage of amyloid fibril formation and under physiologically relevant conditions $(\mathrm{pH}$ and temperature).$^{50,51}$ Furthermore, the local unfolding of $\beta$-domain is considered to be the main trigger for fibril formation. Consistent with previous studies, we observed a similar phenomenon during MD simulations carried out using wildtype HEWL; that is, the localized unfolding of $\beta$-domain appeared to be the key event in inducing fibril formation (Figure 5A-D).

According to Sugimoto and colleagues, ${ }^{52}$ a peptide located in the $\beta$-domain of HEWL referred to as $\mathrm{K}$ peptide (consisting of residues 54-62 GILQINSRW) was found to act as a core for aggregation and subsequent amyloid fibril formation. Likewise, Lara et al. recently found the shorter peptide sequence ILQINS to be highly amyloidogenic at room temperature. ${ }^{53}$ This region of the protein is partially embedded inside the HEWL structure as a pocket in the junction connecting the $\alpha$-domain and the $\beta$ domain. Combined with results from previous studies, our findings in this work indicated that the stability of this region in the $\beta$-domain directly determines the aggregation fate of the entire protein, dictating whether it proceeds to form amorphous aggregates or amyloid fibril. Further understanding of the early events in conformation changes that occur in HEWL unfolding will not only allow a better understanding of the process of HEWL fibril formation but may also enable development of effective ways to inhibit amyloid fibril formation by reducing the ability of HEWL to form locally unfolded intermediates.

Computational approaches to studying how molecules interact are well-established. ${ }^{54,55}$ Applying computational docking studies allowed the exploration of the mechanism of myricetin-induced inhibition of HEWL fibril formation. The smaller blue shift and reduced fluorescence intensity observed by ANS fluorescence assay, an indicator of a less hydrophobic core exposed to solvent, suggested that myricetin could stabilize the whole conformation of HEWL even though it was subjected to fibril-forming conditions (Figure 4A). The docking study revealed that the HEWL binding site for myricetin is located in the region that partly includes the $\mathrm{K}$ peptide located between the $\alpha$-domain and the $\beta$-domain (Figure 6A), the key region associated with amyloid fibril formation. A plausible explanation for the inhibitory action of myricetin is a reduction of locally unfolded intermediates, which in turn will reduce the propensity for amyloid fibril formation. In this binding mode, myricetin may be acting as an adhesive via hydrogen bonding and van der Waals interaction to glue the aggregation-prone region of the $\beta$-domain with the otherwise stable $\alpha$-domain, consequently resulting in a reduced population of transiently $\beta$-domain unfolded intermediates. Thus, no formation of amyloid fibril would occur under this condition. However, further biophysical experimentation is required to fully address the mechanism of fibril inhibition.

Support for the authenticity of the binding site for myricetin, derived from the docking study, is supported by the experimental data from the intrinsic fluorescence assay. Results from the intrinsic fluorescence assay showed that the interaction between the aromatic residues of myricetin and HEWL could account for the quenching of intrinsic fluorescence (Figure 3B). The normalized fluorescence changes versus the different incubation time appeared almost the same for the emission spectra obtained from excitation at 280 and $295 \mathrm{~nm}$ (data not shown), indicating that the quenching of fluorescence can be attributed to Trp residues. Taken together with the fact that Trp62 is one of the two dominant emitters (the other is Trp108), ${ }^{56}$ the binding site which is located close to Trp62, identified by the docking study, is very likely to be the real scenario. This is consistent with previous investigations in which certain small molecules with inhibitory activity toward HEWL fibril formation, such as EGCG, EUK-8, and EUK-134 (two kinds of salen-manganese complexes) were also suggested to bind to Trp62 and Trp63 in docking studies. ${ }^{10,57,58}$ Taken together, our findings provided a plausible rationale by which fibril formation by HEWL could be 
inhibited by small molecules by targeting the aggregation-prone regions in monomers of this amyloidogenic protein.

Human lysozyme is a bacteriolytic enzyme. According to the Phillips mechanism, ${ }^{59}$ the amino acid side chains glutamic acid 35 (Glu35) and aspartate 52 (Asp52) have been found to be critical to the activity of this enzyme. Our docking study predicted that the binding site for myricetin on HEWL was clustered around many residues, such as Asn44, Asn46, Asp52, Asn59 and Glu35, Ala107, Val109, and Trp62 and Trp63. Thus, it is possible that myricetin docking with lysozyme could inhibit its physiological functions to some extent; however, detailed inhibitory studies need to be carried out to determine at what concentration of myricetin is safe and efficient for dietary flavonol consumption or as a drug candidate. It is important to note that fibrillar tangles as well as amorphous aggregates can be cytotoxic; $^{60,61}$ also, a recent study on HEWL amorphous aggregates has demonstrated that $\mathrm{Cu}(\mathrm{II})$ directs formation of toxic amorphous aggregates resulting in inhibition of HEWL fibrillation under alkaline-salt-mediated conditions. ${ }^{62}$ The potential cytotoxicity of amorphous aggregates of HEWL needs to be assessed in detail to fully appreciate the potential of myricetin as a drug candidate.

\section{AUTHOR INFORMATION}

\section{Corresponding Authors}

*(J.H.) E-mail: jwhe@lnu.edu.cn.

*(Y.S.) E-mail: ysong@lnu.edu.cn.

*(G.W.J.) E-mail: gary.jones@nuim.ie.

\section{Funding}

This publication has emanated from research conducted with the financial support of Science Foundation Ireland under the International Strategic Cooperation Award Grant No. SFI/13/ ISCA/2845. This work was supported by grants from the Joint Specialized Research Fund for the Doctoral Program of Higher Education and partially sponsored by the Fund of Science and Technology project (No. F12-277-1-59) from Shenyang Science and Technology Bureau.

\section{Notes}

The authors declare no competing financial interest.

\section{REFERENCES}

(1) Sipe, J. D.; Benson, M. D.; Buxbaum, J. N.; Ikeda, S.; Merlini, G.; Saraiva, M. J.; Westermark, P. Amyloid fibril protein nomenclature: 2012 recommendations from the Nomenclature Committee of the International Society of Amyloidosis. Amyloid 2012, 19 (4), 167-70.

(2) Pepys, M. B.; Hawkins, P. N.; Booth, D. R.; Vigushin, D. M.; Tennent, G. A.; Soutar, A. K.; Totty, N.; Nguyen, O.; Blake, C. C.; Terry, C. J.; et al. Human lysozyme gene mutations cause hereditary systemic amyloidosis. Nature 1993, 362 (6420), 553-7.

(3) Valleix, S.; Drunat, S.; Philit, J. B.; Adoue, D.; Piette, J. C.; Droz, D.; MacGregor, B.; Canet, D.; Delpech, M.; Grateau, G. Hereditary renal amyloidosis caused by a new variant lysozyme W64R in a French family. Kidney Int. 2002, 61 (3), 907-12.

(4) Yazaki, M.; Farrell, S. A.; Benson, M. D. A novel lysozyme mutation Phe57Ile associated with hereditary renal amyloidosis. Kidney Int. 2003, 63 (5), 1652-7.

(5) Rocken, C.; Becker, K.; Fandrich, M.; Schroeckh, V.; Stix, B.; Rath, T.; Kahne, T.; Dierkes, J.; Roessner, A.; Albert, F. W. ALys amyloidosis caused by compound heterozygosity in exon 2 (Thr70Asn) and exon 4 (Trp112Arg) of the lysozyme gene. Human Mutat. 2006, 27 (1), 11920.

(6) Palmer, A. M. Neuroprotective therapeutics for Alzheimer's disease: Progress and prospects. Trends Pharmacol. Sci. 2011, 32 (3), $141-7$.
(7) Cohen, F. E.; Kelly, J. W. Therapeutic approaches to proteinmisfolding diseases. Nature 2003, 426 (6968), 905-9.

(8) Gazit, E. Mechanisms of amyloid fibril self-assembly and inhibition. Model short peptides as a key research tool. FEBS J. 2005, 272 (23), 5971-8.

(9) Porat, Y.; Abramowitz, A.; Gazit, E. Inhibition of amyloid fibril formation by polyphenols: Structural similarity and aromatic interactions as a common inhibition mechanism. Chem. Biol. Drug Des. 2006, 67 (1), 27-37.

(10) Ghosh, S.; Pandey, N. K.; Dasgupta, S. (-)-Epicatechin gallate prevents alkali-salt mediated fibrillogenesis of hen egg white lysozyme. Int. J. Biol. Macromol. 2013, 54, 90-8.

(11) Wang, S. S.; Liu, K. N.; Lee, W. H. Effect of curcumin on the amyloid fibrillogenesis of hen egg-white lysozyme. Biophys. Chem. 2009, $144(1-2), 78-87$.

(12) Vuong, Q. V.; Siposova, K.; Nguyen, T. T.; Antosova, A.; Balogova, L.; Drajna, L.; Imrich, J.; Li, M. S.; Gazova, Z. Binding of glyco-acridine derivatives to lysozyme leads to inhibition of amyloid fibrillization. Biomacromolecules 2013, 14 (4), 1035-43.

(13) Gazova, Z.; Bellova, A.; Daxnerova, Z.; Imrich, J.; Kristian, P.; Tomascikova, J.; Bagelova, J.; Fedunova, D.; Antalik, M. Acridine derivatives inhibit lysozyme aggregation. Eur. Biophys. J. 2008, 37 (7), 1261-70.

(14) Antosova, A.; Chelli, B.; Bystrenova, E.; Siposova, K.; Valle, F.; Imrich, J.; Vilkova, M.; Kristian, P.; Biscarini, F.; Gazova, Z. Structureactivity relationship of acridine derivatives to amyloid aggregation of lysozyme. Biochim. Biophys. Acta 2011, 1810 (4), 465-74.

(15) Wu, J. W.; Liu, K. N.; How, S. C.; Chen, W. A.; Lai, C. M.; Liu, H. S.; Hu, C. J.; Wang, S. S. Carnosine's effect on amyloid fibril formation and induced cytotoxicity of lysozyme. PLoS One 2013, 8 (12), e81982.

(16) Stefani, M.; Rigacci, S. Protein folding and aggregation into amyloid: The interference by natural phenolic compounds. Int. J. Mol. Sci. 2013, 14 (6), 12411-57.

(17) Akaishi, T.; Morimoto, T.; Shibao, M.; Watanabe, S.; Sakai-Kato, K.; Utsunomiya-Tate, N.; Abe, K. Structural requirements for the flavonoid fisetin in inhibiting fibril formation of amyloid beta protein. Neurosci. Lett. 2008, 444 (3), 280-5.

(18) McKoy, A. F.; Chen, J.; Schupbach, T.; Hecht, M. H. A novel inhibitor of amyloid beta (Abeta) peptide aggregation: from high throughput screening to efficacy in an animal model of Alzheimer disease. J. Biol. Chem. 2012, 287 (46), 38992-9000.

(19) Ehrnhoefer, D. E.; Bieschke, J.; Boeddrich, A.; Herbst, M.; Masino, L.; Lurz, R.; Engemann, S.; Pastore, A.; Wanker, E. E. EGCG redirects amyloidogenic polypeptides into unstructured, off-pathway oligomers. Nat. Struct. Mol. Biol. 2008, 15 (6), 558-66.

(20) Bulic, B.; Pickhardt, M.; Schmidt, B.; Mandelkow, E. M.; Waldmann, H.; Mandelkow, E. Development of tau aggregation inhibitors for Alzheimer's disease. Angew. Chem. 2009, 48 (10), $1740-52$.

(21) Ono, K.; Yamada, M. Antioxidant compounds have potent antifibrillogenic and fibril-destabilizing effects for alpha-synuclein fibrils in vitro. J. Neurochem. 2006, 97 (1), 105-15.

(22) Zelus, C.; Fox, A.; Calciano, A.; Faridian, B. S.; Nogaj, L. A.; Moffet, D. A. Myricetin Inhibits Islet Amyloid Polypeptide (IAPP) Aggregation and Rescues Living Mammalian Cells from IAPP Toxicity. Open Biochem. J. 2012, 6, 66-70.

(23) Frare, E.; Polverino De Laureto, P.; Zurdo, J.; Dobson, C. M.; Fontana, A. A highly amyloidogenic region of hen lysozyme. J. Mol. Biol. 2004, 340 (5), 1153-65.

(24) Hess, B.; Kutzner, C.; Van Der Spoel, D.; Lindahl, E. GROMACS 4: Algorithms for highly efficient, load-balanced, and scalable molecular simulation. J. Chem. Theory Comput. 2008, 4, 435-447.

(25) Refaee, M.; Tezuka, T.; Akasaka, K.; Williamson, M. P. Pressuredependent changes in the solution structure of hen egg-white lysozyme. J. Mol. Biol. 2003, 327 (4), 857-65.

(26) Scott, W. R. P.; Hünenberger, P. H.; Tironi, I. G.; Mark, A. E.; Billeter, S. R.; Fennen, J.; Torda, A. E.; Huber, T.; Krüger, P.; van Gunsteren, W. F. The GROMOS biomolecular simulation program package. J. Phys. Chem. A 1999, 103, 3596-607. 
(27) Berendsen, H. J. C.; Postma, J. P. M.; van Gunsteren, W. F.; Hermans, J. Interaction models for water in relation to protein hydration. Intermol. Forces 1981, 11 (Suppl. 1), 331-8.

(28) Essmann, U.; Perera, L.; Berkowitz, M. L.; Darden, T.; Lee, H. J.; Pedersen, L. G. A smooth particle mesh Ewald potential. J. Chem. Phys. 1995, 103, 8577-92.

(29) Chen, C. Y. TCM Database@Taiwan: The world's largest traditional Chinese medicine database for drug screening in silico. PLoS One 2011, 6 (1), No. e15939.

(30) Swaminathan, R.; Ravi, V. K.; Kumar, S.; Kumar, M. V.; Chandra, N. Lysozyme: A model protein for amyloid research. Adv. Protein Chem. Struct Biol. 2011, 84, 63-111.

(31) LeVine, H., 3rd Thioflavine $\mathrm{T}$ interaction with synthetic Alzheimer's disease beta-amyloid peptides: Detection of amyloid aggregation in solution. Protein Sci. 1993, 2 (3), 404-10.

(32) Semisotnov, G. V.; Rodionova, N. A.; Razgulyaev, O. I.; Uversky, V. N.; Gripas, A. F.; Gilmanshin, R. I. Study of the "molten globule" intermediate state in protein folding by a hydrophobic fluorescent probe. Biopolymers 1991, 31 (1), 119-28.

(33) Cowgill, R. W. Fluorescence and the Structure of Proteins. Ii. Fluorescence of Peptides Containing Tryptophan or Tyrosine. Biochim. Biophys. Acta 1963, 75, 272-3.

(34) Di Stasio, E.; Bizzarri, P.; Misiti, F.; Pavoni, E.; Brancaccio, A. A fast and accurate procedure to collect and analyze unfolding fluorescence signal: The case of dystroglycan domains. Biophys. Chem. 2004, 107 (2), 197-211.

(35) Duy, C.; Fitter, J. How aggregation and conformational scrambling of unfolded states govern fluorescence emission spectra. Biophys. J. 2006, 90 (10), 3704-11.

(36) Suryaprakash, P.; Kumar, R. P.; Prakash, V. Thermodynamics of interaction of caffeic acid and quinic acid with multisubunit proteins. Int. J. Biol. Macromol. 2000, 27 (3), 219-28.

(37) Rawel, H. M.; Czajka, D.; Rohn, S.; Kroll, J. Interactions of different phenolic acids and flavonoids with soy proteins. Int. J. Biol. Macromol. 2002, 30 (3-4), 137-50.

(38) Gama Sosa, M. A.; De Gasperi, R.; Elder, G. A. Modeling human neurodegenerative diseases in transgenic systems. Hum. Genet. 2012, 131 (4), 535-63.

(39) Kikis, E. A.; Gidalevitz, T.; Morimoto, R. I. Protein homeostasis in models of aging and age-related conformational disease. Adv. Exp. Med. Biol. 2010, 694, 138-59.

(40) Cuanalo-Contreras, K.; Mukherjee, A.; Soto, C. Role of Protein Misfolding and Proteostasis Deficiency in Protein Misfolding Diseases and Aging. Int. J. Cell Biol. 2013, 2013, No. 638083.

(41) Demontis, F.; Perrimon, N. FOXO/4E-BP signaling in Drosophila muscles regulates organism-wide proteostasis during aging. Cell 2010, 143 (5), 813-25.

(42) Ben-Zvi, A.; Miller, E. A.; Morimoto, R. I. Collapse of proteostasis represents an early molecular event in Caenorhabditis elegans aging. Proc. Natl. Acad. Sci. U. S. A. 2009, 106 (35), 14914-9.

(43) Cohen, E.; Paulsson, J. F.; Blinder, P.; Burstyn-Cohen, T.; Du, D.; Estepa, G.; Adame, A.; Pham, H. M.; Holzenberger, M.; Kelly, J. W.; Masliah, E.; Dillin, A. Reduced IGF-1 signaling delays age-associated proteotoxicity in mice. Cell 2009, 139 (6), 1157-69.

(44) Truelsen, T.; Thudium, D.; Gronbaek, M.; Copenhagen City Heart Study.. Amount and type of alcohol and risk of dementia: The Copenhagen City Heart Study. Neurology 2002, 59 (9), 1313-9.

(45) Ono, K.; Li, L.; Takamura, Y.; Yoshiike, Y.; Zhu, L.; Han, F.; Mao, X.; Ikeda, T.; Takasaki, J.; Nishijo, H.; Takashima, A.; Teplow, D. B.; Zagorski, M. G.; Yamada, M. Phenolic compounds prevent amyloid beta-protein oligomerization and synaptic dysfunction by site-specific binding. J. Biol. Chem. 2012, 287 (18), 14631-43.

(46) Smid, S. D.; Maag, J. L.; Musgrave, I. F. Dietary polyphenolderived protection against neurotoxic beta-amyloid protein: From molecular to clinical. Food Funct. 2012, 3 (12), 1242-50.

(47) Cazarolli, L. H.; Zanatta, L.; Alberton, E. H.; Figueiredo, M. S.; Folador, P.; Damazio, R. G.; Pizzolatti, M. G.; Silva, F. R. Flavonoids: Cellular and molecular mechanism of action in glucose homeostasis. Mini-Rev. Med. Chem. 2008, 8 (10), 1032-8.
(48) Narayana, K. R.; Reddy, M. S.; Chaluvadi, M. R.; Krishna, D. R. Bioflavonoids classification, pharmacological, biochemical effects and therapeutic potential. Indian J. Pharmacol. 2001, 33 (1), 2-16.

(49) Hudson, S. A.; Ecroyd, H.; Kee, T. W.; Carver, J. A. The thioflavin $\mathrm{T}$ fluorescence assay for amyloid fibril detection can be biased by the presence of exogenous compounds. FEBS J. 2009, 276 (20), 5960-72.

(50) Canet, D.; Last, A. M.; Tito, P.; Sunde, M.; Spencer, A.; Archer, D. B.; Redfield, C.; Robinson, C. V.; Dobson, C. M. Local cooperativity in the unfolding of an amyloidogenic variant of human lysozyme. Nat. Struct. Biol. 2002, 9 (4), 308-15.

(51) Dumoulin, M.; Canet, D.; Last, A. M.; Pardon, E.; Archer, D. B.; Muyldermans, S.; Wyns, L.; Matagne, A.; Robinson, C. V.; Redfield, C.; Dobson, C. M. Reduced global cooperativity is a common feature underlying the amyloidogenicity of pathogenic lysozyme mutations. $J$. Mol. Biol. 2005, 346 (3), 773-88.

(52) Tokunaga, Y.; Sakakibara, Y.; Kamada, Y.; Watanabe, K.; Sugimoto, Y. Analysis of core region from egg white lysozyme forming amyloid fibrils. Int. J. Biol. Sci. 2013, 9 (2), 219-27.

(53) Lara, C.; Reynolds, N. P.; Berryman, J. T.; Xu, A.; Zhang, A.; Mezzenga, R. ILQINS hexapeptide, identified in lysozyme left-handed helical ribbons and nanotubes, forms right-handed helical ribbons and crystals. J. Am. Chem. Soc. 2014, 136 (12), 4732-9.

(54) Leach, A. R.; Shoichet, B. K.; Peishoff, C. E. Prediction of proteinligand interactions. Docking and scoring: successes and gaps. J. Med. Chem. 2006, 49 (20), 5851-5.

(55) Kann, M. G. Protein interactions and disease: Computational approaches to uncover the etiology of diseases. Briefings Bioinf. 2007, 8 (5), 333-46.

(56) Imoto, T.; Forster, L. S.; Rupley, J. A.; Tanaka, F. Fluorescence of lysozyme: emissions from tryptophan residues 62 and 108 and energy migration. Proc. Natl. Acad. Sci. U. S. A. 1972, 69 (5), 1151-5.

(57) Bahramikia, S.; Yazdanparast, R. Anti-amyloidogenic and fibrildestabilizing effects of two manganese-salen derivatives against hen eggwhite lysozyme aggregation. Int. J. Biol. Macromol. 2012, 50 (1), 18797.

(58) Kalyan, S. G.; Bijaya, K. S.; Swagata, D. Spectrophotometric studies on the interaction between (-)-epigallocatechin-3-gallate and lysozyme. Chem. Phys. Lett. 2008, 452, 193-7.

(59) Phillips, D. C. The three-dimensional structure of an enzyme molecule. Sci. Am. 1966, 215 (5), 78-90.

(60) Bucciantini, M.; Giannoni, E.; Chiti, F.; Baroni, F.; Formigli, L.; Zurdo, J.; Taddei, N.; Ramponi, G.; Dobson, C. M.; Stefani, M. Inherent toxicity of aggregates implies a common mechanism for protein misfolding diseases. Nature 2002, 416 (6880), 507-11.

(61) Lorenzo, A.; Yankner, B. A. Beta-amyloid neurotoxicity requires fibril formation and is inhibited by congo red. Proc. Natl. Acad. Sci. U. S. A. 1994, 91 (25), 12243-7.

(62) Ghosh, S.; Pandey, N. K.; Banerjee, P.; Chaudhury, K.; Nagy, N. V.; Dasgupta, S. Copper(II) directs formation of toxic amorphous aggregates resulting in inhibition of hen egg white lysozyme fibrillation under alkaline salt-mediated conditions. J. Biomol. Struct. Dyn. 2014, 117. 